\title{
A NOTE ON $H$-SPACES AND POSTNIKOV SYSTEMS OF SPHERES
}

DONALD W. KAHN ${ }^{1}$

In his beautiful paper [1], J. F. Adams settled the question on the nonexistence of maps $S^{2 n-1} \rightarrow S^{n}, n=2^{k}, k>3$, of Hopf invariant one. Taken in conjunction with earlier work, this result shows that the only spheres which are $H$-spaces are $S^{0}, S^{1}, S^{3}$ and $S^{7}$. The present paper grew out of my desire to interpret this result in terms of a Postnikov decomposition for the sphere. There are three sections. The first section contains general results on the Postnikov decompositions of $H$-spaces. The second is devoted entirely to Postnikov decompositions of spheres. In the third section, I consider spaces with simple cohomology, which are thus closely related to spheres. Using the information about Postnikov systems obtained in $\$ 1$, we give some general theorems about these spaces.

Throughout this paper, all spaces will be assumed to have the homotopy-type of a countable, 1-connected complex. All spaces will be assumed to have base points, which will be respected by maps and homotopies. For convenience, we shall omit the base points from the notation.

1. In this section, I collect together several theorems on $H$-spaces. As a starting point, we recall the following theorem, which may be found along with the basic definitions in [3].

Theorem 1.1. Let $X$ and $X^{\prime}$ be spaces and $f: X \rightarrow X^{\prime}$ be a map. Suppose that $X$ and $X^{\prime}$ have Postnikov systems $\left\{X_{n}, P_{n}, \pi_{n, n-1}\right\}$ and $\left\{X_{n}^{\prime}, P_{n}^{\prime}, \pi_{n, n-1}^{\prime}\right\}$. Then, there is a family of maps

$$
f_{n}: X_{n} \rightarrow X_{n}^{\prime}
$$

such that

(a) $\pi_{n+1, n}^{\prime} \cdot f_{n+1}=f_{n} \cdot \pi_{n+1, n}$ and

(b) $P_{n}^{\prime} \cdot f \simeq f_{n} \cdot P_{n}$ for all $n$.

Furthermore, if

$$
k^{n+2} \in H^{n+2}\left(X_{n} ; \pi_{n+1}(X)\right) \quad \text { and } \quad k^{\prime n+2} \in H^{n+2}\left(X_{n}^{\prime} ; \pi_{n+1}\left(X^{\prime}\right)\right)
$$

Received by the editors October 11, 1962 and, in revised form, November 15, 1962.

${ }^{1}$ This work was supported in part by contract NONR $266(57)$ of the Office of Naval Research. 
are k-invariants for these Postnikov systems, we have

$$
f_{n}^{*} k^{\prime n+2}=f_{c}^{t} \cdot k^{n+2}
$$

where $f_{c}^{t}$ is the coefficient homomorphism induced by

$$
f f: \pi_{n+1}(X) \rightarrow \pi_{n+1}\left(X^{\prime}\right) \text {. }
$$

We refer to the maps $f_{n}$ as maps "induced from $f . "$ The maps $f_{n}$ are well-defined, up to homotopy, as is made precise in the following theorem.

Theorem 1.2. Let $\left\{X_{n}, P_{n}, \pi_{n, n-1}\right\}$ and $\left\{X_{n}^{\prime}, P_{n}^{\prime}, \pi_{n, n-1}^{\prime}\right\}$ be Postnikov systems for $X$ and $X^{\prime}$; let $f$ and $g$ be maps from $X$ to $X^{\prime}$. Let $f_{n}$ and $g_{n}$ be the maps induced from $f$ and $g$. If $f \simeq g$, then $f_{n} \simeq g_{n}$, all $n$.

Proof. We have two homotopy commutative diagrams

$$
\begin{aligned}
& X \stackrel{f}{\rightarrow} X^{\prime} \quad X \stackrel{g}{\rightarrow} X^{\prime} \\
& P_{n} \downarrow \quad \downarrow P_{n}^{\prime} \quad P_{n} \downarrow \quad \downarrow P_{n}^{\prime} \\
& X_{n} \underset{f_{n}}{\rightarrow} X_{n}^{\prime} \quad X_{n} \underset{g_{n}}{\rightarrow} X_{n}^{\prime} .
\end{aligned}
$$

By taking a complex of the homotopy-type of $X_{n}$ and forming the fibre space over this complex which is induced from $P_{n}: X \rightarrow X_{n}$, we may replace $X_{n}$ by a complex (same notation). Let $i: X_{n}^{(n+1)} \rightarrow X$ be a cross section over the $(n+1)$-skeleton. We have

$$
\begin{aligned}
f_{n} \mid X_{n}^{(n+1)} & =f_{n} \cdot P_{n} \cdot i\left|X_{n}^{(n+1)} \simeq P_{n}^{\prime} \cdot f \cdot i\right| X_{n}^{(n+1)} \simeq P_{n}^{\prime} \cdot g \cdot i \mid X_{n}^{(n+1)} \\
& \simeq g_{n} \cdot P_{n} \cdot i\left|X_{n}^{(n+1)}=g_{n}\right| X_{n}^{(n+1)} .
\end{aligned}
$$

Thus, $f_{n}$ and $g_{n}$ are homotopic, when restricted to the $(n+1)$-skeleton. We would like to extend this homotopy to all of $X_{n}$. As $\pi_{i}\left(X_{n}^{\prime}\right)=0$, $i>n$, this is clearly possible.

Now, let $X$ be an $H$-space, with multiplication map $\mu: X \times X \rightarrow X$, so that $\mu \cdot i_{j} \simeq I d, j=1,2$, where $i_{j}: X \rightarrow X \times X$ is the inclusion as first or second factor.

Corollary 1.1. If $X$ is an $H$-space, and $\left\{X_{n}, P_{n}, \pi_{n, n-1}\right\}$ is a Postnikov system for $X$, then each $X_{n}$ is an $H$-space.

Proof. By the above theorem, we see that there is a map $\mu_{n}: X_{n}$ $\times X_{n} \rightarrow X_{n}$ such that $\mu_{n} \cdot i_{j} \simeq I d$ (because $\mu \cdot i_{j} \simeq I d$ ).

This corollary was proved in [3] and was also known to Hilton, Ganea, and others. 
For convenience, say that an $H$-space is homotopy invertible, if each element has a homotopy inverse.

Corollary 1.2. Let $X$ be a homotopy $\{$ associative $\},\{$ commutative $\}$, $\{$ invertible $\} H$-space, with Postnikov system $\left\{X_{n}, P_{n}, \pi_{n, n-1}\right\}$. Then each space $X_{n}$ is a homotopy $\{$ associative $\},\{$ commutative $\},\{$ invertible $\}$ $H$-space.

Proof. Suppose $X$ is homotopy commutative. Then the diagram

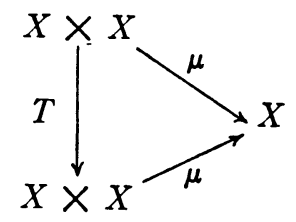

is homotopy commutative, where the map $T$ interchanges the factors. By the above theorem, we have $\mu_{n} \cdot T_{n} \simeq(\mu \cdot T)_{n} \simeq \mu_{n}$, which shows that $X_{n}$ is homotopy commutative.

The other cases follow in a similar way.

In what is to follow, we shall need a characterization of $H$-spaces in terms of homotopy groups and $k$-invariants. Suppose that $\left\{X_{n}, P_{n} ; \pi_{n, n-1}\right\}$ is a Postnikov system, and the space $X_{n}$, for some $n$, is an $H$-space. We say that $k^{n+2} \in H^{n+2}\left(X_{n}, \pi_{n+1}(X)\right)$ is primitive, if $\sigma \cdot \mu_{n}^{*}\left(k^{n+2}\right)=0$, where $\mu_{n}$ is the multiplication on $X_{n}$ and $\sigma$ is the projection onto the second factor in the decomposition

$$
\begin{aligned}
& H^{n+2}\left(X_{n} \times X_{n} ; \pi_{n+1}(X)\right) \\
& \quad=H^{n+2}\left(X_{n} \vee X_{n} ; \pi_{n+1}(X)\right) \oplus H^{n+2}\left(X_{n} \times X_{n}, X_{n} \vee X_{n} ; \pi_{n+1}(X)\right)
\end{aligned}
$$

Theorem 1.3. (a) If $X$ is an $H$-space, with Postnikov decomposition $\left\{X_{n}, P_{n}, \pi_{n, n-1}\right\}$, then each $k$-invariant $k^{n+2} \in H^{n+2}\left(X_{n}, \pi_{n+1}(X)\right)$ is primitive with respect to the induced multiplication $\mu_{n}$.

(b) If each of the k-invariants of a Postnikov system $\left\{X_{n}, P_{n}, \pi_{n, n-1}\right\}$ for $X$ are primitive with respect to the (inductively constructed) $H$-space structures on the $\left\{X_{n}\right\}$, then $X$ is an $H$-space.

Proof. (a) is proved in $\$ 3$ of [3]. (b) is proved in [3], in the case when $X$ has only finitely-many nonvanishing homotopy groups. The proof, in the general case, is obtained by passing to the semi-simplicial case (taking singular complexes), and observing that in that case, $X$ is of the homotopy-type of the projective limit of the $X_{n}$. As each $X_{n}$ is an $H$-space and all the maps $\pi_{n, n-1}$ respect the multiplications (i.e., are $H$-maps), we see that a complex, which is equivalent to $X$, admits a multiplication. As $X$ is assumed to have the 
homotopy-type of a countable complex, the realization of the multiplication gives the desired $H$-space structure.

2. We now specialize to Postnikov decompositions of spheres.

Theorem 2.1. (a) The first $(m-1)$-nontrivial terms in any Postnikov decomposition $\left\{X_{n}, p_{n}, \pi_{n, n-1}\right\}$ of the $m$-sphere, $S^{m}, m>1$, are $H$-spaces.

(b) If $m=3,7$, all terms in any Postnikov decomposition are $H$ spaces. (In fact, in both cases they are all homotopy invertible $H$-spaces, while for $m=3$, they are all also homotopy associative $H$-spaces.)

Proof. To prove (a) we shall show that

$$
H^{m+i}\left(X_{m+i-2} \times X_{m+i-2}, X_{m+i-2} \bigvee X_{m+i-2} ; \pi_{m+i-1}\left(S^{m}\right)\right)=0
$$

for $2 \leqq i \leqq m-1$. It will then follow that $\sigma \cdot \mu_{m+i-2}\left(k^{m+i}\right)=0$ in this range, and we may then apply Theorem 1.3. Now, by the universal coefficient theorem, it is sufficient to show that

$$
H_{j}\left(X_{m+i-2} \times X_{m+i-2}, X_{m+i-2} \bigvee X_{m+i-2} ; Z\right)=0
$$

for $m<j \leqq m+i$. But then it is sufficient to show that the homology of the pair vanishes for coefficients in any field, $K$. But by the Kunneth theorem,

$$
\begin{aligned}
H_{j}\left(X_{m+i-2} \times X_{m+i-2}, X_{m+i-2}\right. & \left.\vee X_{m+i-2} ; K\right) \\
& \cong \sum_{r+s=j ; r, s \neq 0} H_{r}\left(X_{m+i-2} ; K\right) \otimes H_{s}\left(X_{m+i-2} ; K\right) .
\end{aligned}
$$

As $X_{m+i-2}$ has the same $(m+i-2)$-type as $S^{m}$, we have

$$
H_{r}\left(X_{m+i-2} ; K\right)= \begin{cases}0, & 0<r<m, \\ K, & r=m, \\ 0, & m<r \leqq m+i-2 .\end{cases}
$$

Therefore, in the above decomposition of the $j$ th homology group as a tensor product, in order that say the first factor be nonzero, it must have dimension $m$, and then the second factor will be zero.

Part (b) follows immediately from Corollary 1.1 and Corollary 1.2.

We will now show that if $m>1, m \neq 3,7$, then $k^{2 m}$ in a Postnikov decomposition of $S^{m}$ is not primitive. Actually, the following stronger theorem holds.

THEOREM 2.2. Let $X$ be an $(m-1)$-connected space satisfying $H^{r}\left(X ; Z_{2}\right)= \begin{cases}Z_{2}, & j=m, \text { and } H^{i}\left(X ; Z_{p}\right)=0, \quad p \text { odd }, \quad m \leqq i \leqq 2 m, \\ 0, & m<j \leqq 2 m .\end{cases}$ 
If $m>7$, then $X$ is not an $H$-space. In fact, if $\left\{X_{n}, P_{n}, \pi_{n, n-1}\right\}$ is a Postnikov decomposition for $X, X_{2 m-1}$ is not an $H$-space.

Proof. We first show that if $X_{2 m-1}$ were an $H$-space, then it would be a space of loops. We make use of the well-known fact that if a fibre space, with fibre an Eilenberg-MacLane space, and base a space of loops, is such that the $k$-invariant (image of the fundamental class under transgression) is an image under suspension in the loop space, then the fibre space is itself a space of loops.

If $X_{2 m-1}$ were an $H$-space, then by Theorem 3.1 all the $k$-invariants, $k^{i}, m+2 \leqq i \leqq 2 m$, would be primitive. If on each successive term, $X_{m+i}, 1 \leqq i \leqq m-1$, the multiplication was, up to homotopy, the same as the multiplication as a loop space, then the conclusion that $X_{2 m-1}$ is a space of loops will follow by induction. ${ }^{2}$

Hence, we show that at each stage, $X_{m+i}, 1 \leqq i \leqq m-1$, there is a unique such multiplication on $X_{m+i}$, up to homotopy. First note that the multiplication is unique on the $m$-skeleton, because it is multiplicatively of the same $m$-type as an Eilenberg-MacLane space, which has a unique multiplication. Now, observe that any obstruction to deforming one multiplication into another must lie in

$$
H^{i}\left(X_{m+i} \times X_{m+i} ; \pi_{j}\left(X_{m+i}\right)\right) .
$$

If $j>m+i$, then the coefficients are zero. On the other hand, it is easy to see that

$$
H_{j}\left(X_{m+i} \times X_{m+i} ; Z_{2}\right)=0, \quad m<j \leqq m+i
$$

and $H_{m}\left(X_{m+i} \times X_{m+i} ; Z\right)$ is free. Thus,

$$
H^{i}\left(X_{m+i} \times X_{m+i} ; \pi_{j}\left(X_{m+i}\right)\right)
$$

is zero in all dimensions greater than $m$. Hence, we may construct the desired deformation of one multiplication into the other.

We have shown that $X_{2 n-1}$ is a space of loops, assuming that it was an $H$-space. That means that there is a contractible fibre space ( $\left.E, X_{2 m-1}, B\right)$, with $X_{2 m-1}$ as fibre. In the Serre spectral sequence [5] of this fibre space, with $Z_{2}$ coefficients, one has the element $u \otimes x$ $\in E_{2}^{m+1, m}$, which is the tensor product of the (mod 2) fundamental classes in the base and fibre. Since it is clear that $H^{j}\left(X_{2 m-1} ; Z_{2}\right)=0$, $m<i \leqq 2 m$, we see that in order for $u \otimes x$ not to remain until $E_{\infty}$, we must have $u^{2} \neq 0$ in the base. Note that $H^{i}\left(B ; Z_{2}\right)=0, m+1<j<2 m$ +2 . According to Adams [1], such a cohomology ring is impossible, if $m+1>8$. Hence, $X_{2 m-1}$ could not have been an $H$-space.

2 All $k$-invariants in this range must necessarily be suspensions. 
Returning to $S^{m}, m>7$, the above shows that in any Postnikov decomposition, $k^{2 m}$ is not a primitive element in $H^{2 m}\left(X_{2 m-2} ; \pi_{2 m-1}\left(S^{m}\right)\right)$, with respect to the unique $H$-space structure on $X_{2 m-2}$. It would be of interest, of course, to determine $k^{2 m}$ exactly. However, as $\pi_{2 m-1}\left(S^{m}\right)$ is unknown in general, this is at present impossible. We can obtain some information, when the coefficients are in $Z_{2}$.

Proposition 2.1. With the above notations, let $k \in H^{2 m}\left(X_{2 m-2} ; Z_{2}\right)$ be a nonprimitive element. If $k$ is the $\bmod 2$ reduction of $a$ class $\bar{k} \in H^{2 m}\left(X_{2 m-2} ; Z\right)$ then $\bar{k}$ has infinite order.

PROOF. If $\bar{k}$ had order $m$, then we would have $\mu_{2 m-2}^{*}(m \bar{k})$ equal to $m(x \otimes x)$, where $x \in H^{m}\left(X_{2 m-2} ; Z\right)$ is the fundamental class, and this clearly has infinite order. ${ }^{3}$

REMARK. Using the results of Adams [1], it is possible to construct an explicit nonprimitive element in $H^{2 m}\left(X_{2 m-2} ; Z_{2}\right)$, for the case $m=2^{K}-1, K>3$. For other values of $m \neq 3,7$, such elements may be constructed using the Adem relations. In either case, the method consists of constructing a nonprimitive element in what is sort of a universal example. In the dimension range which we need, an element is nonprimitive, if it is not a suspension.

REMARK. If it were possible to define multiplications on the terms $X_{i}, i \geqq 2 m-1$, in a decomposition of a sphere, the corresponding $k$ invariants would also be forced to be primitive by the cohomology of the sphere. Thus, in some sense, the question of whether a sphere can be an $H$-space is decided by the element $k^{2 m}$.

3. Let $L(\pi, m), m>1$, denote a co-Moore space, i.e., a (1-connected) space such that

$$
H^{i}(L(\pi, m) ; Z)= \begin{cases}Z, & i=0 \\ 0, & 0<i<m \\ \pi, & i=m \\ 0, & m<i .\end{cases}
$$

Such a space is a sort of generalization of a sphere. It is not the case that such a space exists for any $\pi$ and $m$. If $\pi=Q=$ rational numbers, it has long been known that such a space cannot exist. (See, for example [2].) I now will give some direct applications of the preceding results to these spaces. For simplicity, we shall only consider the crude cases, that is we shall prove certain theorems about the nonvanishing of homotopy groups. A more refined approach might be used to discuss the orders of certain elements.

\footnotetext{
${ }^{3}$ Of course, this elementary proposition holds for any finite field of coefficients.
} 
THEOREM 3.1. Let $\pi$ be a nontrivial, countable, torsion-free Abelian group, for which there exists a space $X=L(\pi, m)$. Then $X$ is $(m-1)$ connected and $\pi_{m}(X)$ is nonzero.

If $X$ is not an $H$-space, then $\pi_{2 m-1}(X)$ is nonzero. (In other words, if, for such a space, $\pi_{2 m-1}(X)=0$, then $X$ is an $H$-space.)

Proof. Since $\pi$ is torsion-free, the first part of the theorem follows from the Hurewicz theorem and the universal coefficient theorem.

Suppose $X$ is not an $H$-space. If in a Postnikov system $\left\{X_{n}, p_{n}, \pi_{n, n-1}\right\}$ for $X$, the space $X_{2 n-1}$ were an $H$-space, then, as remarked above, all the higher $k$-invariants would be forced to be primitive by the simple cohomology of $X$. This would make $X$ an $H$-space, so that $X_{2 m-1}$ cannot be an $H$-space, and $k^{2 m}$ must be nonprimitive with respect to some multiplications on $X_{2 m-2}$. In particular, $k^{2 m}$ is nonzero, and $\pi_{2 m-1}(X) \neq 0$.

REMARK. Using results of J. P. Meyer [4], one can show even more; there is a nontrivial Whitehead product lying in dimension $2 n-1$. This generalizes the well-known result that if $S^{n}$ is not an $H$-space, $\left[i_{n}, i_{n}\right] \neq 0$, where $i_{n}$ is a generator of $\pi_{n}\left(S^{n}\right)$.

We will now prove an extension of the previous theorem to the case where $\pi$ is an arbitrary countable group for which there exists a space $L(\pi, n)$.

TheOREM 3.2. Let $\pi$ be a countable group, for which there exists a space $L(\pi, n)$, with $n>3$. If $X=L(\pi, n)$ is not an $H$-space, then one of the homotopy groups

$$
\pi_{2 n-1}(X), \quad \pi_{2 n-2}(X), \quad \pi_{2 n-3}(X), \quad \pi_{2 n-4}(X)
$$

must be nontrivial.

Proof. The space $X$ is clearly $(n-2)$-connected. We consider the relative homology group

$$
H_{i}\left(X_{n+j} \times X_{n+j}, X_{n+j} \vee X_{n+j} ; Z\right),
$$

where $X_{n+j}$ is a space in a Postnikov decomposition for $X$. If this group $\left({ }^{*}\right)$ is nontrivial for some $i$ and $j$, then there exists a field $K$ such that the group

$$
\sum_{r+s=i ; r, s \neq 0} H_{r}\left(X_{n+j} ; K\right) \otimes_{K} H_{s}\left(X_{n+j} ; K\right)
$$

is nonzero. Now the group $H_{r}\left(X_{n+j} ; K\right)$ will be nonzero, for any $K$, only if $r=n, n-1$ or $r>n+j$. Hence, the group $\left(^{*}\right)$ can be nonzero, 
only if $i=2 n, 2 n-1,2 n-2$, or $2 n-3$, provided $i<2 n+j-1$. It follows that the group

$$
H^{n+i+2}\left(X_{n+j} \times X_{n+j}, X_{n+j} \bigvee X_{n+j} ; \pi_{n+j+1}(X)\right)
$$

can be nonzero, only if $n+j+2=2 n, 2 n-1,2 n-2,2 n-3$.

To prove the theorem, note that by Theorem 1.3, some $k$-invariant $k^{n+j+2}$ must be nonprimitive, with respect to an $H$-space structure on $X_{n+j}$. We have shown that this is possible, only when $n+j+2=2 n$, $2 n-1,2 n-2$, or $2 n-3$. Hence, one of the homotopy groups which are listed in the statement of the theorem must not vanish.

REMARK. In the case of Theorem 3.2, one may obtain some partial information about Whitehead products.

One may use these techniques to prove similar theorems about spaces with one nonvanishing, integral homology group. (For any prescribed group, such a space always exists.) Of course, one may apply these arguments to more complicated spaces, but naturally the results are much more complicated to state.

\section{BIBLIOGRAPHY}

1. J. F. Adams, On the non-existence of elements of Hopf invariant one, Ann. of Math. (2) 72 (1960), 20-104.

2. D. M. Kan and G. Whitehead, On the realizability of singular cohomology groups, Proc. Amer. Math. Soc. 12 (1961), 24-25.

3. D. W. Kahn, Induced maps for Postnikov systems, Trans. Amer. Math. Soc. 107 (1963), 432-450.

4. J. P. Meyer, Whitehead products and Postnikov systems, Amer. J. Math. 82 (1960), 271-280.

5. J.-P. Serre, Homologie singulière des espaces fibrés, Ann. of Math. (2) 54 (1951), 425-505.

Columbia University 